This is a "preproof" accepted article for The Bulletin of Symbolic Logic. This version may be subject to change during the production process.

DOI: $10.1017 /$ bsl.2021.29

\title{
Sealing of the universally Baire sets ${ }^{* \dagger}$
}

\author{
Grigor Sargsyan $¥$ \\ Nam Trang $\S$
}

October 26,2019

\begin{abstract}
A set of reals is universally Baire if all of its continuous preimages in topological spaces have the Baire property. Sealing is a type of generic absoluteness condition introduced by Woodin that asserts in strong terms that the theory of the universally Baire sets cannot be changed by set forcings.

The Largest Suslin Axiom (LSA) is a determinacy axiom isolated by Woodin. It asserts that the largest Suslin cardinal is inaccessible for ordinal definable surjections. Let LSA-over-uB be the statement that in all (set) generic extensions there is a model of LSA whose Suslin, co-Suslin sets are the universally Baire sets.

We outline the proof that over some mild large cardinal theory, Sealing is equiconsistent with LSA-over-uB. In fact, we isolate an exact theory (in the hierarchy of strategy mice) that is equiconsistent with both (see Definition 3.1). As a consequence, we obtain that Sealing is weaker than the theory "ZFC+there is a Woodin cardinal which is a limit of Woodin cardinals". This significantly improves upon the earlier consistency proof of Sealing by Woodin. A variation of Sealing, called Tower Sealing, is also shown to be equiconsistent with Sealing over the same large cardinal theory.

We also outline the proof that if $V$ has a proper class of Woodin cardinals, a strong cardinal, and a generically universally Baire iteration strategy then

*2000 Mathematics Subject Classifications: 03E15, 03E45, 03E60.

${ }^{\dagger}$ Keywords: Mouse, inner model theory, descriptive set theory, hod mouse.

${ }^{\ddagger}$ Department of Mathematics, Rutgers University, NJ, USA. Email: gs481@math.rutgers.edu

$\S$ Department of Mathematics, University of North Texas, Denton, TX, USA. Email:

"Revised February 8, 2021 and April 9, 2021.
\end{abstract} Nam.Trang@unt.edu 
Sealing holds after collapsing the successor of the least strong cardinal to be countable. This result is complementary to the aforementioned equiconsistency result, where it is shown that Sealing holds in a generic extension of a certain minimal universe. This theorem is more general in that no minimal assumption is needed. A corollary of this is that LSA-over-uB is not equivalent to Sealing.

We identify elements of the Baire space $\omega^{\omega}$ with reals. Throughout the paper, by a "set of reals $A$ ", we mean $A \subseteq \omega^{\omega}$. Given a cardinal $\kappa$, we say $T \subseteq \bigcup_{n<\omega} \omega^{n} \times \kappa^{n}$ is a tree on $\omega \times \kappa$ if $T$ is closed under initial segments. Given a tree $T$ on $\omega \times \kappa$, we let $[T]$ be the set of its branches, i.e., $b \in[T]$ if $b \in \omega^{\omega} \times \kappa^{\omega}$ and letting $b=\left(b_{0}, b_{1}\right)$, for each $n \in \omega,\left(b_{0}\left\lceil n, b_{1}\lceil n) \in T\right.\right.$. We then let $p[T]=\left\{x \in \omega^{\omega}: \exists f((x, f) \in[T])\right\}$. A set of reals $A$ is $\gamma$-universally Baire if there are trees $T, U$ on $\omega \times \lambda$ for some $\lambda$ such that $A=p[T]=\mathbb{R} \backslash p[U]$ and whenever $g$ is $<\gamma$-generic (i.e. $g$ is $V$-generic for some forcing $\mathbb{P} \in V$ such that $|\mathbb{P}|<\gamma)$, in $V[g], p[T]=\mathbb{R} \backslash p[U]$. We write $A^{g}$ for $p[T]^{V[g]}$; this is the canonical interpretation of $A$ in $V[g] .{ }^{1} A$ is universally Baire if $A$ is $\gamma$-universally Baire for all $\gamma$. Let $\Gamma^{\infty}$ be the set of universally Baire sets. Given a generic $g$, we let $\Gamma_{g}^{\infty}=\left(\Gamma^{\infty}\right)^{V[g]}$ and $\mathbb{R}_{g}=\mathbb{R}^{V[g]}$. The notion of universal Baireness was first isolated and studied in [FMW92]. ${ }^{2}$ A proper class of Woodin cardinals is typically assumed when studying universally Baire sets as it ensures $\Gamma^{\infty}$ behaves nicely, e.g. $\Gamma^{\infty}$ is closed under real quantifiers, continuous substitutions, and any two sets in $\Gamma^{\infty}$ are Wadge comparable under this assumption.

The discovery of forcing almost immediately initiated the study of removing independence phenomena from set theory. Large cardinals were used to establish a plethora of results that generalize Shoenfield's Absoluteness Theorem to more complex formulas than $\Sigma_{2}^{1}$. Sealing is a perhaps the strongest generalization of Shoenfield's Absoluteness Theorem one could hope for.

Definition 0.1 (Woodin) Sealing is the conjunction of the following statements.

1. For every set generic $g, L\left(\Gamma_{g}^{\infty}, \mathbb{R}_{g}\right) \vDash \mathrm{AD}^{+}$and $\wp\left(\mathbb{R}_{g}\right) \cap L\left(\Gamma_{g}^{\infty}, \mathbb{R}_{g}\right)=\Gamma_{g}^{\infty}$.

2. For every set generic $g$ over $V$, for every set generic $h$ over $V[g]$, there is an elementary embedding

$$
j: L\left(\Gamma_{g}^{\infty}, \mathbb{R}_{g}\right) \rightarrow L\left(\Gamma_{g * h}^{\infty}, \mathbb{R}_{g * h}\right)
$$

such that for every $A \in \Gamma_{g}^{\infty}, j(A)=A^{h}$.

\footnotetext{
${ }^{1}$ One can show $A^{g}$ does not depend on the choice of $T, U$.

${ }^{2}$ One can find a proof that the two definitions of universal Baireness that we mention in this paper are equivalent.
} 
Woodin showed that if $A$ is a universally Baire set of reals and the universe has a proper class of Woodin cardinals then the theory of $L(A, \mathbb{R})$ cannot be changed by set forcings. He achieved this by showing that if there is a proper class of Woodin cardinals then for any universally Baire set $A$ and any two successive set generic extensions $V[g] \subseteq V[h]$, there is an elementary embedding $j: L\left(A_{g}, \mathbb{R}_{g}\right) \rightarrow L\left(A_{h}, \mathbb{R}_{h}\right)$. Under the stated large cardinal assumption, all $\Sigma_{2}^{1}$ sets of reals are universally Baire. In fact, the largest class of sets of reals for which a Shoenfield-type generic absoluteness can hold is the collection of the universally Baire sets in the sense that if sufficient generic absoluteness is true about a set of reals then that set is universally Baire. More precisely, suppose $\phi$ is a property of reals. Let $A_{\phi}$ be the set of reals defined by $\phi$. If sufficiently many statements about $A_{\phi}$ are generically absolute then it is because $A_{\phi}$ is universally Baire (see [Ste09, Lemma 4.1]). Thus, the next place to look for absoluteness is the set of all universally Baire sets.

The existence of a proper class of Woodin cardinals does not imply Sealing. In fact, in the minimal mouse with a proper class of Woodin cardinals, clause (1) of Sealing fails. Woodin, [Lar04], has shown that assuming there exist a proper class of Woodin cardinals and a supercompact cardinal, letting $\delta$ be a supercompact cardinal, then in $V^{\text {Coll }\left(\omega, V_{\delta+1}\right)}$, Sealing holds. Theorem 1.1 shows that Sealing's consistency strength is below that of the existence of a Woodin cardinal which is a limit of Woodin cardinals, i.e. within the short extender region. The proof of this fact is beyond the scope of this paper; roughly, it follows from recent unpublished work of the first author, which shows that excellent hybrid mice, defined in 3.1, exist, assuming the existence of divergent models of $\mathrm{AD}^{+}$; and the latter is consistent relative to the existence of a Woodin cardinal which is a limit of Woodin cardinals. ${ }^{3}$ Therefore, Sealing is not a strong consequence of supercompactness as suggested by Woodin's theorem.

Recent works, [ST19b],[ST19c], suggest that Sealing has significant impacts on various aspects of inner model theory and the inner model program. A more detailed discussion of these connections is given in [ST19b].

To introduce LSA-over-uB, we first need to introduce the Largest Suslin Axiom (LSA). A cardinal $\kappa$ is OD-inaccessible if for every $\alpha<\kappa$ there is no surjection $f: \wp(\alpha) \rightarrow \kappa$ that is definable from ordinal parameters. A set of reals $A \subseteq \mathbb{R}$ is $\kappa$-Suslin if for some tree $T$ on $\omega \times \kappa, A=p[T]$. A set $A$ is Suslin if it is $\kappa$-Suslin for

\footnotetext{
${ }^{3}$ Another outline of the proof that Sealing is consistent relative to the existence of a Woodin cardinal which is a limit of Woodin cardinals is as follows. The existence of an lbr hod premouse $\mathcal{P}$ as in [Sar20, Theorem 1.2] follows from the existence of a Woodin limit of Woodin cardinals by [Sar20, Step 4]. Then letting $\lambda_{0}$ be as in [Sar20, Theorem 1.2], $\mathcal{P} \mid \lambda_{0}$ satisfies the hypothesis of [ST19c, Theorem 0.4], which is Theorem 2.2 stated in this paper.
} 
some $\kappa ; A$ is co-Suslin if its complement $\mathbb{R} \backslash A$ is Suslin. A set $A$ is Suslin, co-Suslin if both $A$ and its complement are Suslin. A cardinal $\kappa$ is a Suslin cardinal if there is a set of reals $A$ such that $A$ is $\kappa$-Suslin but $A$ is not $\lambda$-Suslin for any $\lambda<\kappa$. Suslin cardinals play an important role in the study of models of determinacy (see for example, various articles from the Cabal Volumes: [KMM83], [KMS88], [KLS08], [KLS12], [KLS16], [KMM81], [KM78]).

The Largest Suslin Axiom was introduced by Woodin in [Woo10, Remark 9.28]. The terminology is due to the first author. Here is the definition.

Definition 0.2 The Largest Suslin Axiom, abbreviated as LSA, is the conjunction of the following statements:

1. $\mathrm{AD}^{+}$.

2. There is a largest Suslin cardinal.

3. The largest Suslin cardinal is OD-inaccessible.

In the hierarchy of determinacy axioms, which one may appropriately call the Solovay Hierarchy ${ }^{4}$, LSA is an anomaly as it belongs to the successor stage of the Solovay Hierarchy but does not conform to the general norms of the successor stages of the Solovay Hierarchy. LSA is a very strong determinacy axiom; for example, it implies there are models of " $A D_{\mathbb{R}}+\Theta$ is regular". Prior to [ST], LSA was not known to be consistent. In [ST], the first author showed that it is consistent relative to a Woodin cardinal that is a limit of Woodin cardinals. Nowadays, the axiom plays a key role in many aspects of inner model theory, and features prominently in Woodin's Ultimate L framework (see [Woo17, Definition 7.14] and Axiom I and Axiom II on page 97 of $\left.\left[\mathrm{Woo}_{17}\right]^{5}\right)$.

Definition 0.3 Let LSA-over-uB be the statement: For all $V$-generic $g$, in $V[g]$, there is $A \subseteq \mathbb{R}_{g}$ such that $L\left(A, \mathbb{R}_{g}\right) \vDash \mathrm{LSA}$ and $\Gamma_{g}^{\infty}$ is the Suslin co-Suslin sets of $L\left(A, \mathbb{R}_{g}\right)$.

\footnotetext{
${ }^{4}$ Solovay defined what is now called the Solovay Sequence (see [Woo10, Definition 9.23]). It is a continuous sequence of ordinals with the largest element $\Theta$, where $\Theta$ is the least ordinal that is not a surjective image of the reals. One then obtains a hierarchy of axioms by requiring that the Solovay Sequence has complex patterns. LSA is an axiom in this hierarchy. The reader may consult [Sar13] or [Woo10, Remark 9.28].

${ }^{5}$ The requirement in these axioms that there is a strong cardinal which is a limit of Woodin cardinals is only possible if $L(A, \mathbb{R}) \vDash$ LSA.
} 
LSA-over-uB is isolated by the authors in [ST19b] as part of the consistency calculations of Sealing. LSA-over-uB plays a role in clarifying relationships between strong forcing axioms such as Martin's Maximum (MM) and variations of Woodin's (*)-axiom. For example, Woodin has observed, after the second author's talk on the topic of Sealing at the XVI International Workshop in Set Theory at CIRM, Luminy, that assuming there is a proper class of Woodin cardinals, then MM + LSA-over-UB implies $(*)^{++}$fails, where $(*)^{++}$, which says that $\wp(\mathbb{R})$ belongs to a $\mathbb{P}_{\text {max }}$-extension of a determinacy model containing all reals and ordinals, is a strengthening of $(*)$, which says that $\wp\left(\omega_{1}\right)$ belongs to a $\mathbb{P}_{\text {max }}$-extension of $L(\mathbb{R})$, the minimal model of determinacy containing all reals and ordinals. This suggests that $\mathrm{MM}^{++}$cannot imply $(*)^{++}$, in contrast to a recent result of Aspero and Schindler that $\mathrm{MM}^{++}$ implies $(*)$.

Acknowledgement. The authors would like to thank the NSF for its generous support. The first author is supported by NSF Career Award DMS-1352034. The second author is supported by NSF Grants DMS-1565808, DMS-1849295, and NSF Career Grant DMS-1945592. We would also like to thank the referee for a careful read of a previous version of the paper and for offering several helpful suggestions that improve the exposition.

\section{The consistency of Sealing}

The following is our main theorem. We say that $\phi$ and are equiconsistent over theory $T$ if there is a model of $T \cup\{\phi\}$ if and only if there is a model of $T \cup\{\psi\}$.

Theorem 1.1 ([ST19b]) Sealing and LSA-over-uB are equiconsistent over "there exists a proper class of Woodin cardinals and the class of measurable cardinals is stationary".

The following variation of Sealing, called Tower Sealing, is a stronger statement than the version isolated by Woodin ( [Lar04]). Tower Sealing turns out to be equiconsistent with Sealing and LSA-over-uB over the base theory of Theorem 1.1.

Definition 1.2 Tower Sealing is the conjunction of:

1. For any set generic $g, L\left(\Gamma_{g}^{\infty}\right) \vDash \mathrm{AD}^{+}$, and $\Gamma_{g}^{\infty}=\wp(\mathbb{R}) \cap L\left(\Gamma_{g}^{\infty}, \mathbb{R}_{g}\right)$.

2. There is a proper class of Woodin cardinals and for any set generic $g$, in $V[g]$, suppose $\delta$ is Woodin, whenever $G$ is $V[g]$-generic for either the $\mathbb{P}_{<\delta}$-stationary tower or the $\mathbb{Q}_{<\delta}$-stationary tower at $\delta$, then 


$$
j\left(\Gamma_{g}^{\infty}\right)=\Gamma_{g * G}^{\infty},
$$

where $j: V[g] \rightarrow M \subset V[g * G]$ is the generic elementary embedding given by $G$.

Woodin has observed that assuming a proper class of Woodin cardinals which are limits of strong cardinals, Tower Sealing implies Sealing. The converse is not known. However, we can show

Theorem 1.3 ([ST19b]) Tower Sealing and Sealing are equiconsistent over "there exists a proper class of Woodin cardinals and the class of measurable cardinals is stationary".

Woodin, in [Lar04], shows Sealing and a weak form of Tower Sealing are consistent relative to the existence of a supercompact cardinal and a proper class of Woodin cardinals. As explained above, a corollary of the theorems above, and recent work in [Ste16] and [Sar], is the following theorem, which significantly improves Woodin's theorem. Throughout this paper, we let WLW denote the theory "ZFC + there is a Woodin cardinal which is a limit of Woodin cardinals".

Theorem 1.4 Con(WLW) implies Con(Sealing + Tower Sealing).

In fact, the proof Theorem 1.4 shows that in a generic extension of an excellent hybrid mouse, cf. Definition 3.1, both Sealing and Tower Sealing hold. As mentioned in the introduction, the consistency of the existence of an excellent hybrid mouse follows from Con(WLW).

\section{Self-iterability and Sealing}

Next, we state another theorem about the consistency of Sealing that implies Sealing and LSA-over-uB are not equivalent. The Unique Branch Hypothesis (UBH) is the statement that every normal iteration tree $\mathcal{T}$ on $V$ has at most one cofinal wellfounded branch. The Generic Unique Branch Hypothesis (gUBH) says that UBH holds in all set generic extensions. The notions of a pre-iterable structure and a generically universally Baire (guB) strategy are discussed in detail in [ST19c, Section 1].

Suppose $P$ is a transitive model of set theory. We let ile $(P)$ be the set of inaccessible-length extenders of $P$. More precisely ile $(P)$ consists of extenders $E \in P$ such that $P \vDash " l h(E)$ is inaccessible and $V_{l h(E)}=V_{l h(E)}^{U l t(V, E)}$ ", here $l h(E)$ is the length of the extender $E$. 
Definition 2.1 We say that self-iterability holds if the following holds in $V$.

1. gUBH.

2. $\mathcal{V}=(V$, ile $(V))$ is a pre-iterable structure that has a guB-iteration strategy.

The notions in Definition 2.1 abstract some of the essential properties of universally Baire strategies of fine-structural extender models that we need for the proof of the following theorem.

Theorem 2.2 ([ST19c]) Assume self-iterability holds, and suppose there is a proper class of Woodin cardinals and a strong cardinal. Let $\kappa$ be the least strong cardinal of $V$ and let $g \subseteq \operatorname{Coll}\left(\omega, \kappa^{+}\right)$be $V$-generic. Then $V[g] \vDash$ Sealing.

Remark 2.3 (i) Normal iteration trees on $\mathcal{V}$ are coarse and are plus-2 trees in the sense of [MS94]. However, the hypothesis of Theorem 2.2 cannot be weakened to just gUBH for plus-2 iterations as this form of UBH holds in a minimal mouse with a strong cardinal, a class of Woodin cardinals and a stationary class of measurable cardinals ${ }^{6}$, but this theory is weaker than Sealing as shown by the proof of Theorem 1.1 .

(ii) We note that there is no anti-large cardinal assumption in Theorem 2.2. Contrast this with the situation in the previous section, where Sealing is shown to hold in a generic extension of a minimal universe of a certain theory (i.e. the universe of a minimal excellent hybrid mouse, see Definition 3.1).

(iii) The proof of Theorem 2.2 is accessible to those with general knowledge of iterations, iteration strategies, and Woodin's extender algebra, all of which are topics that can be presented without any fine structure theory.

Recall from [Ste16] the statement of Hod Pair Capturing (HPC): For any Suslin co-Suslin set $A \subseteq \mathbb{R}$, there is a least-branch (lbr) hod pair $(\mathcal{P}, \Sigma)$ such that $A$ is definable from parameters over $(H C, \in, \Sigma)$. No Long Extender (NLE) is the statement: there is no countable, $\omega_{1}+1$-iterable pure extender premouse $M$ such that there is a long extender on the $M$-sequence. The notion of least-branch hod mice (lbr hod mice) is defined precisely in [Ste16, Section 5].

Definition 2.4 gHPC is the statement: suppose $V[g]$ is a set generic extension of $V$, suppose in $V[g], M=L(\Gamma, \mathbb{R})$ is a model of $\mathrm{AD}^{+}$, then $M \vDash \mathrm{HPC}$.

\footnotetext{
${ }^{6}$ This fact is due to Steel, see [Ste07, Theorem 3.3].
} 
Theorem 2.5 ([ST19c]) Suppose self-iterability holds and there is a proper class of inaccessible cardinals which are limit of Woodin cardinals. Suppose gHPC and NLE hold. Then $V \vDash$ LSA-over-uB fails.

Remark 2.6 (i) The proof of this theorem is given in [ST19c, Section 7]. One shows that letting $\lambda$ be an inaccessible cardinal which is a limit of Woodin cardinals and $g \subseteq \operatorname{Coll}(\omega,<\lambda)$ be $V$-generic, then in $V[g]$, there cannot be a set $A \subset \mathbb{R}$ such that $L(A, \mathbb{R}) \vDash \mathrm{LSA}$ and $\Gamma^{\infty}$ is the Suslin-co-Suslin sets of $L(A, \mathbb{R})$.

(ii) The hypotheses of Theorem 2.5 hold in the universe of lbr hod mice that have a proper class of inaccessible cardinals which are limit of Woodin cardinals (cf. [Ste07, Theorem 3.3] and [Ste16]). So such hod mice satisfy "LSA-over-uB fails."

Remark 2.6, Theorem 2.5, and the fact that self-iterability and gHPC hold in any generic extension of an lbr hod mouse with a proper class of Woodin cardinals give us the following.

Corollary 2.7 ([ST19c]) Let $V$ be the universe of an lbr hod mouse with a proper class of inaccessible cardinals which are limit of Woodin cardinals and a strong cardinal. Let $\kappa$ be the least strong cardinal of $V$ and $g \subseteq \operatorname{Coll}\left(\omega, \kappa^{+}\right)$be $V$-generic. Then $V[g] \vDash$ "Sealing holds and LSA-over-uB fails".

Corollary 2.7 is surprising and unexpected in light of the above results. For example, generic absoluteness for $L(\mathbb{R})$, namely, for all successive generics $g$ and $h$ there is an elementary embedding $j: L\left(\mathbb{R}_{g}\right) \rightarrow L\left(\mathbb{R}_{g * h}\right)$, is equivalent to the existence and the universal Baireness of the next canonical set beyond $L(\mathbb{R})$, namely $\mathbb{R}^{\# 7}$. While one cannot hope that Sealing would imply both the existence and the universal Baireness of the next canonical set of reals beyond $\Gamma^{\infty 8}$, one could still hope that the cause of Sealing is the existence of some nice set of reals just like the cause of the generic absoluteness of $L(\mathbb{R})$ is the universal Baireness of $\mathbb{R}^{\# 9}$. Because the next nice set beyond $\Gamma^{\infty}$ cannot be universally Baire, the best we can hope for is that the next set beyond $\Gamma^{\infty}$ creates an LSA model over $\Gamma^{\infty}$. In fact, this discussion was the original motivation for isolating LSA-over-UB. Contrary to our expectations, what causes Sealing to hold may not be coded into a set of reals as demonstrated by Corollary 2.7.

\footnotetext{
${ }^{7}$ This fact is due to Steel and Woodin. For example, see genericity iterations in [Ste10].

${ }^{8}$ As all universally Baire sets are already in $\Gamma^{\infty}$.

${ }^{9}$ Or rather the universal Baireness of the $\omega_{1}$-iteration strategy of $\mathcal{M}_{\omega}^{\#}$.
} 


\section{Outline of proofs}

We first give a brief outline of the proofs of Theorems 1.1 and 1.3. The crucial notion involved in these proofs is that of an excellent hybrid premouse, which is a kind of short-tree strategy hybrid premice, where the short-tree strategy is that of an lsa hod premouse. The notions of lsa hod premice and their short-tree strategies are crucial for the analysis of models of LSA and are treated in detail in [ST]. Roughly an lsa hod premouse $\mathcal{P}$ is a hod premouse with the largest Woodin cardinal $\delta^{\mathcal{P}}, \mathcal{P}=\left(\mathcal{P} \mid \delta^{\mathcal{P}}\right)^{\sharp}$, and letting $\kappa$ be the least $<\delta^{\mathcal{P}}$-strong cardinal in $\mathcal{P}$, then $\kappa$ is a limit of Woodin cardinals. If $\Sigma$ is an iteration strategy of $\mathcal{P}$, then the short part $\Sigma^{s h}$ of $\Sigma$ is the part of $\Sigma$ that acts on short trees according to $\Sigma$, so $\Sigma^{s h}(\mathcal{T})$ is defined and is $\Sigma(\mathcal{T})$ if and only if $\mathcal{T}$ is short. ${ }^{10}$ We say that $(\mathcal{P}, \Lambda)$ is a short-tree strategy (sts) hod pair if $\mathcal{P}$ is an lsa hod premouse and $\Lambda$ is a short-tree strategy of $\mathcal{P}$. If $\mathcal{Q}$ is a $\Lambda$-premouse above $\mathcal{P}$, then we say that $\mathcal{Q}$ is an sts premouse based on $\mathcal{P} .{ }^{11}$

Suppose $(\mathcal{P}, \Lambda)$ is a sts hod pair, where $\Lambda$ has hull condensation and strong branch condensation (see [ST] for a detailed discussion of these notions). $\Lambda$ has branch condensation for pullbacks if whenever $\pi: \mathcal{Q} \rightarrow \mathcal{P}$ is elementary, the $\pi$-pullback of $\Lambda$ has branch condensation. For a more detailed discussion of these topics, see [Sar15], [ST], and [ST19b].

We say that $[\nu, \delta]$ is a window if there are no Woodin cardinals in the interval $(\nu, \delta)$. Given a window $w$, we let $\nu^{w}$ and $\delta^{w}$ be such that $w=\left[\nu^{w}, \delta^{w}\right]$. We say that window $w$ is above $\kappa$ if $\nu^{w} \geq \kappa$. Suppose $\kappa$ is a cardinal. We say window-based self-iterability (WBSI) holds at $\kappa$ if for any window $w$ that is above $\kappa$ and for any successor cardinal $\eta \in\left(\nu^{w}, \delta^{w}\right)$, setting $Q=H_{\eta^{+}}, Q$ has an $O r d$-iteration strategy $\Sigma$ which acts on iterations that only use extenders with critical points $>\nu^{w}$.

Definition 3.1 Suppose $\mathcal{P}$ is hybrid premouse. We say that $\mathcal{P}$ is excellent if

1. $\mathcal{P} \vDash T_{0}$, where $T_{0}$ says "ZFC + There is a proper class of Woodin cardinals + the class of measurable cardinals is stationary + no measurable cardinal that is a limit of Woodin cardinals carries a normal ultrafilter concentrating on the set of measurable cardinals."

\footnotetext{
${ }^{10}$ One can let $\Sigma^{s h}(\mathcal{T})$ be undefined or be equal to $\mathcal{M}(\mathcal{T})^{\sharp}$ as in [ST]. One major technical challenge of analyzing the HOD of the minimal model of LSA is to define short tree strategies without having the full strategies available and indexing short trees in lsa hod mice. See [ST] for more details.

${ }^{11}$ Again, the reader can consult [ST] for details on how to index short trees and the $\mathcal{Q}$-structure authentication procedure in $\mathcal{Q}$. This is one major technical advance that [ST] contributes to the study of hod mice.
} 
2. There is a Woodin cardinal $\delta$ of $\mathcal{P}$ such that $\mathcal{P} \vDash " \mathcal{P}_{0}={ }_{\text {def }}(\mathcal{P} \mid \delta)^{\#}$ is a hod premouse of lsa type", $\mathcal{P}$ is an sts premouse based on $\mathcal{P}_{0}$ and $\mathcal{P} \vDash " S^{\mathcal{P}}$, which is a short tree strategy for $\mathcal{P}_{0}$, has hull condensation, strong branch condensation, and branch condensation for pull-backs".

3. Given any $\tau<\delta^{\mathcal{P}_{0}}$ such that $\left(\mathcal{P}_{0} \mid \tau\right)^{\#}$ is of lsa type, there is $\mathcal{M} \triangleleft \mathcal{P}$ such that $\tau$ is a cutpoint of $\mathcal{M}$ and $\mathcal{M} \vDash ~ " \tau$ is not a Woodin cardinal".

4. Letting $\delta$ be as above, $\mathcal{P} \vDash$ "WBSI holds at $\delta "$.

If $\mathcal{P}$ is excellent then we let $\delta^{\mathcal{P}}$ be the $\delta$ of clause 2 above and $\mathcal{P}_{0}=\left(\left(\mathcal{P} \mid \delta^{\mathcal{P}}\right)^{\#}\right)^{\mathcal{P}} .12$

For one direction of Theorems 1.1 and 1.3, assume the existence of an excellent hybrid premouse $\mathcal{P}$. Let $\delta=\delta^{\mathcal{P}}, \kappa$ be the least $<\delta$-strong cardinal in $\mathcal{P}_{0}$, and $g \subset \operatorname{Coll}(\omega, \delta)$ be $\mathcal{P}$-generic. Then one can show in $\mathcal{P}[g]$, Sealing, Tower Sealing, and LSA-over-uB hold. We give a sketch of Sealing holds; the reader can consult [ST19b, Section 3] for a detailed proof. Let $\Lambda$ be the short-tree strategy of $\mathcal{P}_{0}$ coded by $S^{\mathcal{P}}$. The smallness assumptions in clause (1) of Definition 3.1 amongst other things imply that all local non-Woodin cardinals of a hod premouse (or hybrid premouse) are witnessed by $\mathcal{Q}$-structures which are initial segments of $\mathcal{P}$ and are tame. Clause (3) prevents us from constructing a "smaller" excellent hybrid premouse by performing certain fully-backgrounded sts constructions over $\left(\mathcal{P}_{0} \mid \tau\right)^{\sharp}$ inside $\mathcal{P}$. Clauses $(2)$ and (4) (combined with clause (1)) allow us to extend $\Lambda$ and the window-based strategy for $\mathcal{P} \mid \eta$, where $\eta \in\left[\nu^{w}, \delta^{w}\right]$ and $\left[\nu^{w}, \delta^{w}\right]$ is a window above $\delta$, to any set generic extension over $\mathcal{P}$ (cf. [ST19b, Section 2]. Let $h$ be a generic filter over $\mathcal{P}[g]$ and $\Lambda^{h}$ be the interpretation of $\Lambda$ in $\mathcal{P}[g * h]$. We then can "capture" $\Gamma_{g * h}^{\infty}$ in the following sense: letting $\Delta^{h}$ be the collection of $A \subseteq \mathbb{R}^{\mathcal{P}[g * h]}$ in $\mathcal{P}[g * h]$ such that for some countable $\mathcal{T}$ according to $\Lambda^{h}$ such that the iteration embedding restricted to $\mathcal{P}_{0}^{b}=\left(\mathcal{P}_{0} \mid\left(\kappa^{+}\right)^{\mathcal{P}}\right)$ exists, then

$$
\Delta^{h}=\Gamma_{g * h}^{\infty} .
$$

[ST19b, Lemma 3.3] shows that $L\left(\Delta^{h}, \mathbb{R}^{\mathcal{P}[g * h]}\right)$ can be realized as the derived model of an iterate of $\mathcal{P}_{0}$. The proof of [ST19b, Lemma 3.3] and various lemmas in [ST19b, Section 2] uses substantially the fact that window-based iterability in $\mathcal{P}$ and its generic extensions implies various backgrounded constructions in $\mathcal{P}$ and in its generic extensions converge. Equation (1) then implies clause (1) of Sealing. A standard argument, cf [ST19b, Lemma 3.4], shows that for any generic filter $k$ over $\mathcal{P}[g * h]$, there is an elementary embedding $j: L\left(\Delta^{h}, \mathbb{R}^{\mathcal{P}[g * h]}\right) \rightarrow L\left(\Delta^{h * k}, \mathbb{R}^{\mathcal{P}[g * h * k]}\right)$ such that for any $A \in \Delta^{h}, j(A)=A^{k}$. This implies clause (2) of Sealing.

\footnotetext{
${ }^{12}$ We allow for the possibility that an excellent hybrid premouse is a proper class.
} 
The proof of Theorem 2.2's main idea is similar to the sketch above. The selfiterability hypothesis of the theorem for the most part allows us to simulate the proof sketched above. We can find a transitive model $R^{13}$ with sufficient iterability such that $H_{\kappa^{+}} \subset R$ and there is an iterate $S$ of $R$ such that for some $\lambda$, a limit of Woodin cardinals of $S$, for some $S$-generic $g \subseteq \operatorname{Coll}(\omega,<\lambda)$, letting $\mathbb{R}_{g}^{*}=\bigcup_{\alpha<\lambda} \mathbb{R}^{S[g \cap \operatorname{Coll}(\omega, \alpha)] \text {, }}$ and $H o m_{g}^{*}$ be the collection of sets $A \in S\left(\mathbb{R}_{g}^{*}\right) \cap \wp(\mathbb{R})$ such that for some $\alpha<\lambda$, there is a pair $(T, U) \in S[g \cap \operatorname{Coll}(\omega, \alpha)]$ such that $S[g \cap \operatorname{Coll}(\omega, \alpha)] \vDash "(T, U)$ are $<\lambda$-absolutely complementing trees" and $p[T]^{V\left(\mathbb{R}^{*}\right)}=A$, then we have the following:

- $\mathbb{R}_{g}^{*}=\mathbb{R}^{V}$.

- $\operatorname{Hom}_{g}^{*}=\Gamma^{\infty}$.

The fact that $\kappa$ is strong is used substantially in the proof of $H o m_{g}^{*}=\Gamma^{\infty}$ above. Sealing holds in $V^{\operatorname{Coll}\left(\omega, \kappa^{+}\right)}$then follows by a standard argument. See [ST19c].

Now we sketch the proof of the other direction of Theorems 1.1 and 1.3. In particular, assuming Sealing (or Tower Sealing, or LSA-over-uB) and the large cardinal base theory of the theorems, we want to show that excellent hybrid premice exist. The proof of this occupies Sections 4-10 of [ST19b] and involves many details and ideas. Many of these ideas, especially the notion of condensing sets and various hybrid (sts) backgrounded constructions, feature prominently in the HOD analysis of models of LSA as well as the core model induction proof that LSA is consistent relative to PFA, cf. [ST]. The main thing we need to prove is: assuming no excellent hybrid premice exist, then certain hybrid (sts) fully backgrounded constructions converge (see [ST19b, Sections 8-10]) to a model $\mathcal{P}$, which turns out to be an excellent hybrid premouse. There are many consequences that we need from the hypothesis to guarantee the convergence of such constructions, but one main set of consequences is described in [ST19b, Section 5]: there is a stationary class $S$ of measurable cardinals $\mu$ which is a limit of Woodin cardinals, there is a $\nu<\min (S)$, and there are sequences $\left(Y_{\mu}: \mu \in S\right),\left(A_{\mu}: \mu \in S\right)$ such that the following hold for $\mu \in S$ :

(i) letting $h \subset \operatorname{Coll}(\omega,<\mu)$ be $V$-generic, then $H_{o} m_{h}^{*}=\Gamma_{h}^{\infty}$,

(ii) $A_{\mu} \in Y_{\mu}$ and $A_{\mu}$ codes $H_{\mu}$, and the hod limit of $L\left(H o m_{h}^{*}, \mathbb{R}_{h}^{*}\right),{ }^{14}$

(iii) whenever $Y_{\mu} \subseteq X \prec H_{\mu^{+}}$and $X$ has size $<\mu$, is closed under $\nu$-sequences, $X$ captures $\operatorname{Lp}^{c u B}, \Psi_{\mu}\left(A_{\mu}\right)$ in the sense that letting $\pi_{X}: M_{X} \rightarrow X$ be the uncollapse

\footnotetext{
${ }^{13} R$ can be taken to be the transitive collapse of a sufficiently elementary substructure of $V_{\xi}$, for some large, regular cardinal $\xi$.

${ }^{14}$ The hod limit does not depend on the choice of generics.
} 
map, then $\pi_{X}^{-1}\left(L p^{c u B, \Psi_{\mu}}\left(A_{\mu}\right)\right)=L p^{c u B, \Lambda}\left(\pi_{X}^{-1}\left(A_{\mu}\right)\right)$, where $\Lambda$ is the $\pi_{X}$-pullback strategy of $\Psi_{\mu} \cdot{ }^{15}$

Sealing (or Tower Sealing, or LSA-over-uB) and the fact that $\mu$ is measurable can be used to show

$$
\operatorname{cof}\left(o\left(L p^{c u B, \Psi_{\mu}}\left(A_{\mu}\right)\right)\right)<\mu .
$$

The significance of $\nu$ is that it is an ordinal that bounds $\operatorname{cof}\left(o\left(L p^{c u B, \Psi_{\mu}}\left(A_{\mu}\right)\right)\right)$ for all $\mu \in S$ and $\nu$ is obtained from the usual Fodor's argument applied to the (class) function $\mu \mapsto \operatorname{cof}\left(o\left(L p^{c u B, \Psi_{\mu}}\left(A_{\mu}\right)\right)\right)$; this in turns allows us to "capture" $L p^{c u B, \Psi_{\mu}}\left(A_{\mu}\right)$ by a club of $X$ as described in (iii) above. The reader can consult [ST19b] for details on how these facts are put together into a proof of convergence of various hybrid fully backgrounded constructions.

\section{Variations of Sealing and open problems}

The rather mild assumption used in Theorems 1.1 and 1.3 that the class of measurable cardinals is stationary is probably not needed. As mentioned above, it is mainly used in [ST19b, Section 5] to show that a certain (definable) regressive class function is constant on a stationary class of measurable cardinals. The existence of the ordinal $\nu$ above follows from this. We suspect that refinements of our techniques will eliminate this assumption. We conjecture the following.

Conjecture 4.1 The following theories are equiconsistent.

1. Sealing + "There is a proper class of Woodin cardinals".

2. LSA-over-uB + "There is a proper class of Woodin cardinals".

3. Tower Sealing + "There is a proper class of Woodin cardinals".

As discussed in [ST19b], the core model induction becomes very difficult past Sealing. A good test problem for practitioners of the core model induction is.

Problem 4.2 Prove that Con(PFA) implies Con(WLW).

\footnotetext{
${ }^{15}$ Here $\Psi_{\mu}$ is the strategy of $\mathcal{H}_{\mu}$, the canonical one-cardinal extension of the hod limit of $L\left(H_{o m}^{*}, \mathbb{R}_{h}^{*}\right)$ and the stack $\mathrm{Lp}^{c u B, \Psi}$ is defined in [ST19b, Section 4].
} 
We know from the results above that WLW is stronger than Sealing and is roughly the strongest natural theory at the limit of traditional methods for proving iterability. We believe it is plausible to develop core model induction methods for obtaining canonical models of WLW from just PFA. ${ }^{16}$ The paper [ST19a] is the first step towards this goal; in the paper, we have constructed from PFA hod mice that are stronger than $\mathcal{P}$ in Definition 3.1.

The following are variations of the theories discussed above.

Definition 4.3 Sealing ${ }^{+}$is the conjunction of:

1. For every set generic $g$ over $V$, in $V[g], \wp\left(\mathbb{R}_{g}\right) \cap L\left(\Gamma_{g}^{\infty}, \mathbb{R}_{g}\right)=\Gamma_{g}^{\infty}$ and $L\left(\Gamma_{g}^{\infty}, \mathbb{R}_{g}\right) \vDash$ "AD $\mathbb{R}_{\mathbb{R}}+\Theta$ is regular".

2. For every set generic $g$ over $V$, for every set generic $h$ over $V[g]$, in $V[g][h]$, there is an elementary embedding

$$
j: L\left(\Gamma_{g}^{\infty}, \mathbb{R}_{g}\right) \rightarrow L\left(\Gamma_{g * h}^{\infty}, \mathbb{R}_{g * h}\right)
$$

such that for every $A \in \Gamma_{g}^{\infty}, j(A)=A^{h}$.

Sealing- is the statement: "For every set generic $g$ over $V$, in $V[g], \Gamma_{g}^{\infty}=\wp(\mathbb{R}) \cap$ $L\left(\Gamma_{g}^{\infty}, \mathbb{R}_{g}\right)$ and there is no $\omega_{1}$ sequence of distinct reals in $L\left(\Gamma_{g}^{\infty}, \mathbb{R}_{g}\right)$."

LSA-over-uB- is the statement: "For every set generic $g$ over $V$, in $V[g]$, there is $A \subseteq \mathbb{R}_{g}$ such that $L\left(A, \mathbb{R}_{g}\right) \vDash \mathrm{LSA}$ and $\Gamma_{g}^{\infty}$ is contained in $L\left(A, \mathbb{R}_{g}\right)$."

The proofs of Theorems 1.1 and 1.3 show the following. Let $T=$ "there exists a proper class of Woodin cardinals and the class of measurable cardinals is stationary". Then the following theories are equiconsistent:

1. Sealing $+T$.

2. Sealing ${ }^{+}+T$.

3. Sealing ${ }^{-}+\mathrm{T}$.

\footnotetext{
${ }^{16}$ The second author observes, cf. [ST19a], that assuming PFA and there is a Woodin cardinal, then there is a canonical model of WLW. The proof is not via core model induction methods, but just an observation that the full-backgrounded construction as done in [Nee02] reaches a model of WLW. The Woodin cardinal assumption is important here. The argument would not work if one assumes just PFA and/or a large cardinal milder than a Woodin cardinal, e.g. a measurable cardinal or a strong cardinal.
} 


\section{Tower Sealing $+\mathrm{T}$.}

5. LSA-over-uB $+\mathrm{T}$.

6. LSA-over-uB $^{-}+\mathrm{T}$.

The following conjecture, if true, would be an ultimate analog of the main result of [Ste02], which shows, assuming a proper class of measurable cardinals, the statement "AD holds in $L(\mathbb{R})^{V^{\mathbb{P}}}$ for every poset $\mathbb{P}$ " is equivalent to "for every poset $\mathbb{P}, L(\mathbb{R})^{V^{\mathbb{P}}} \vDash$ there is no uncountable sequence of distinct reals".

Conjecture 4.4 Suppose there is a proper class of Woodin cardinals and the class of measurable cardinals is stationary. Then the following are equivalent.

1. Sealing.

2. Sealing ${ }^{+}$.

3. Sealing ${ }^{-}$.

4. Tower Sealing.

We end this paper by the following question. In every known model of MM+ there is a proper class of Woodin cardinals, $(*)^{++}$fails.

Question 4.5 1. Is $\mathrm{MM}+\mathrm{LSA}$-over-UB consistent?

2. Is $\mathrm{MM}+(*)^{++}$consistent?

\section{References}

[FMW92] Qi Feng, Menachem Magidor, and Hugh Woodin, Universally Baire sets of reals, Set theory of the continuum (Berkeley, CA, 1989), Math. Sci. Res. Inst. Publ., vol. 26, Springer, New York, 1992, pp. 203-242. MR 1233821

[KLS08] Alexander S. Kechris, Benedikt Löwe, and John R. Steel (eds.), Games, scales, and Suslin cardinals. The Cabal Seminar. Vol. I, Lecture Notes in Logic, vol. 31, Association for Symbolic Logic, Chicago, IL; Cambridge University Press, Cambridge, 2008. MR 2463612 
[KLS12] Alexander S. Kechris, Benedikt Löwe, and John R. Steel (eds.), Wadge degrees and projective ordinals. The Cabal Seminar. Volume II, Lecture Notes in Logic, vol. 37, Association for Symbolic Logic, La Jolla, CA; Cambridge University Press, Cambridge, 2012. MR 2906066

[KLS16] Alexander S. Kechris, Benedikt Löwe, and John R. Steel (eds.), Ordinal definability and recursion theory. The Cabal Seminar. Vol. III, Lecture Notes in Logic, vol. 43, Association for Symbolic Logic, Ithaca, NY; Cambridge University Press, Cambridge, 2016, Including reprinted papers from the Caltech-UCLA Cabal seminars held in Los Angeles, CA. MR 3410197

[KM78] Alexander S. Kechris and Yiannis N. Moschovakis (eds.), Cabal Seminar 76-77, Lecture Notes in Mathematics, vol. 689, Springer, Berlin, 1978. MR 526912

[KMM81] Alexander S. Kechris, Donald A. Martin, and Yiannis N. Moschovakis (eds.), Cabal Seminar 77-79, Lecture Notes in Mathematics, vol. 839, Springer, Berlin, 1981. MR 611165

[KMM83] A. S. Kechris, D. A. Martin, and Y. N. Moschovakis (eds.), Cabal seminar 79-81, Lecture Notes in Mathematics, vol. 1019, Springer-Verlag, Berlin, 1983. MR 730583

[KMS88] A. S. Kechris, D. A. Martin, and J. R. Steel (eds.), Cabal Seminar 81-85, Lecture Notes in Mathematics, vol. 1333, Springer-Verlag, Berlin, 1988. MR 960892

[Lar04] Paul B. Larson, The stationary tower, University Lecture Series, vol. 32, American Mathematical Society, Providence, RI, 2004, Notes on a course by W. Hugh Woodin. MR 2069032

[MS94] D. A. Martin and J. R. Steel, Iteration trees, J. Amer. Math. Soc. 7 (1994), no. 1, 1-73. MR 1224594

[Nee02] Itay Neeman, Inner models in the region of a Woodin limit of Woodin cardinals, Ann. Pure Appl. Logic 116 (2002), no. 1-3, 67-155. MR MR1900902 (2003e:03100)

[Sar] Grigor Sargsyan, Generic generators, to appear.

[Sar13] _ Descriptive inner model theory, Bull. Symbolic Logic 19 (2013), no. $1,1-55$. MR 3087400 
[Sar15] G. Sargsyan, Hod mice and the mouse set conjecture, vol. 236, Memoirs of the American Mathematical Society, no. 1111, American Mathematical Society, 2015.

[Sar20] Grigor Sargsyan, Announcement of recent results in descriptive inner model theory, available http://www.grigorsargis.net/.

[ST] Grigor Sargsyan and Nam Trang, The Largest Suslin Axiom, Submitted. Available at math.rutgers.edu/ gs481/lsa.pdf.

[ST19a] _ A core model induction past the Largest Suslin Axiom, In preparation.

[ST19b] _ The exact consistency strength of generic absoluteness for universally Baire sets, In preparation.

[ST19c] S S S _ S Laling from iterability, to appear on the Transactions of the American Mathematical Society, available at math.unt.edu/ ntrang (2019).

[Ste02] John R Steel, Core models with more Woodin cardinals, The Journal of Symbolic Logic 67 (2002), no. 03, 1197-1226.

[Ste07] John R. Steel, Local $K^{c}$ constructions, The Journal of Symbolic Logic 72 (2007), no. 3, 721-737.

[Ste09] J. R. Steel, The derived model theorem, Logic Colloquium 2006, Lect. Notes Log., vol. 32, Assoc. Symbol. Logic, Chicago, IL, 2009, pp. 280327. MR 2562557

[Ste10] John R Steel, An outline of inner model theory, Handbook of set theory (2010), 1595-1684.

[Ste16] John R. Steel, Normalizing iteration trees and comparing iteration strategies, Available at math.berkeley.edu/ steel/papers/Publications.html.

[Woo10] W. Hugh Woodin, The axiom of determinacy, forcing axioms, and the nonstationary ideal, revised ed., De Gruyter Series in Logic and its Applications, vol. 1, Walter de Gruyter GmbH \& Co. KG, Berlin, 2010. MR 2723878

[Woo17] In search of Ultimate-L: the 19th Midrasha Mathematicae Lectures, Bull. Symb. Log. 23 (2017), no. 1, 1-109. MR 3632568 